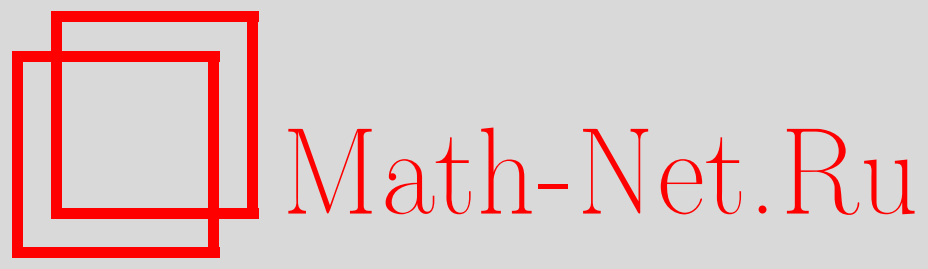

А. М. Вершик, А. В. Малютин, Фазовый переход в задаче о границе-выход для случайных блужданий на группах, Функи. анализ и его прил., 2015, том 49, выпуск 2, 7-20

DOI: https://doi.org/10.4213/faa3195

Использование Общероссийского математического портала MathNet.Ru подразумевает, что вы прочитали и согласны с пользовательским соглашением

http://www . mathnet.ru/rus/agreement

Параметры загрузки:

IP : 54.198 .67 .100

26 апреля 2023 г., 16:44:39

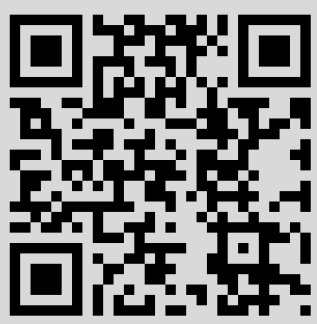




\title{
Фазовый переход в задаче о границе-выход для случайных блужданий на группах
}

\author{
(c) 2015. А. М. ВеРшик, А. В. МАлютин
}

Памяти Е. Б. Дынкина

\begin{abstract}
В работе описывается полная граница-выход случайных блужданий на однородных деревьях, в том числе на свободных группах. В этой модели существует фазовый переход, состоящий в потере эргодичности семейства марковских мер при изменении параметра случайного блуждания.

Рассматриваемая проблема является частным случаем задачи об инвариантных (центральных) мерах на графах ветвления, охватывающей целый ряд проблем комбинаторики, теории представлений, теории вероятностей и в полном объеме поставленной в серии недавних работ первого автора [1]-[3]. С другой стороны, близкие задачи в контексте теории марковских процессов обсуждались еще в 60-х годах Е. Б. Дынкиным.
\end{abstract}

\section{1. Введение}

В конце 60-х гг. Е. Б. Дынкин в статьях [9]-[11] развил концепцию границвыход (соответственно границ-вход) $)^{1)}$ для марковских цепей с заданными копереходными (соответственно переходными) вероятностями. Он отправлялся в основном от теории Мартина и ее вероятностной интерпретации, данной Дж. Дубом, Дж. Хантом и др. В книге [8] изложен ряд задач этой теории. Например, описана граница Мартина для естественного оператора Лапласа на свободной группе, т. е. множество минимальных гармонических функций. В книге [19] собраны многочисленные результаты о различных границах классических симметрических пространств.

На связь этих задач с задачей отыскания инвариантных (центральных) мер на пространстве путей графов ветвления (т. е. локально конечных счетных связных $\mathbb{N}$-градуированных графов), или диаграмм Браттели, неоднократно указывалось в ряде работ первого автора и С. В. Керова. В недавней работе [3] была отчетливо сформулирована равнооблемность задачи о поиске границывыход, т.е. поиске множества всех марковских мер с данными копереходными вероятностями, и задачи об описании множества мер с заданным коииклом (в случае чентральных мер кочикл тождественно равен 1) для гиперконечного отношения эквивалентности на канторовском множестве. Как подчеркнуто в [3], наиболее прозрачным и геометричным контекстом для этих задач является теория проективных пределов симплексов. С другой стороны, самые важные факты, обнаруженные недавно [3], связаны со свойствами хвостовых фильтраций марковского процесса (или с пространством путей графа ветвления), такими, как стандартность и компактность во внутренней метрике.

*Работа поддержана грантом РНФ 14-11-00581.

1) По терминологии этих работ - пространств выходов (входов). 
В данной статье мы рассматриваем частную задачу, а именно - задачу о поиске границы-выход для простых случайных блужданий на деревьях или, эквивалентным образом, задачу описания центральных мер для графов ветвления, связанных с этими случайными блужданиями. В дальнейших работах будет рассмотрен более широкий класс групп и графов ветвления.

Опишем конструкцию динамических графов - графов ветвления, в терминах которых исследуется задача. Пусть $T$ - произвольный связный локально конечный граф без кратных ребер с отмеченной вершиной. Динамический граб $\mathrm{D}(T)$ графа $T$ определяется как $\mathbb{N}$-градуированный граф, $n$-й этаж которого является копией множества тех вершин графа $T$, которые соединены с отмеченной вершиной маршрутами длины $n$ в $T$ (или, что то же самое, путями длины не более $n$ и той же, что и $n$, четности в $D(T))$; единственную вершину нулевого этажа обозначим через $\varnothing$. Две вершины в $\mathrm{D}(T)$ являются соседними, если и только если они расположены на соседних уровнях и соединены ребром в графе $T$. Если связный граф $T$ не имеет нечетных циклов, то выбор начальной вершины превращает его в $\mathbb{N}$-градуированный граф, для которого динамический граф D $(T)$ определен в [5] и назван паскализацией графа $T^{1)}$.

Если $G$ - счетная группа с фиксированным конечным набором образующих $A=A^{-1}$, то под динамическим графом $\mathrm{D}(G, A)$ пары $(G, A)$ понимается динамический граф $\mathrm{D}(T(G, A))$, где $T(G, A)$ есть граф Кэли пары $(G, A)$; граф $\mathrm{D}(G, A)$ мы называем динамическим графом Кэли ${ }^{2)}$.

В этой работе в качестве графа $T$ мы будем всегда рассматривать однородное дерево $T_{q+1}, q>0$, валентности $q+1$. Граф $T_{2}$ есть граф Кэли группы $\mathbb{Z}$, граф $T_{2 k}$ есть граф Кэли свободной группы с $k$ образующими относительно множества образующих и обратных к ним; см. рис. 1, 2.

Напомним теперь общее определение центральных и эргодических мер на пространстве путей произвольного графа ветвления D (см. [3]). Введем канторовское множество $\mathscr{P}(\mathrm{D})$ всех бесконечных путей в графе D. Это компакт в слабой топологии. Мы будем рассматривать борелевские вероятностные меры на этом пространстве. Мера $\mu$ на $\mathscr{P}(\mathrm{D})$ называется иентральной, если она обладает следующим свойством: для любой вершины $v$ графа D условная мера на множестве конечных путей, соединяющих $\varnothing$ и вершину $v$, равномерна (т. е. равна единице, деленной на число таких путей). Нетрудно видеть, что всякая центральная мера является марковской относительно градуировки, т. е. законом марковской цепи, для которой номера этажей - это моменты времени от 0 до $+\infty^{3)}$. Легко проверить, что все центральные меры имеют одну и ту же систему копереходных вероятностей, т. е. копереходная вероятность попасть из произвольной вершины $u$ этажа $n$ в фиксированную вершину $v$ этажа $n+1$ пропорциональна числу путей, ведущих из $\varnothing$ в вершину $u$.

1) Термин связан с тем, что для цепи (т. е. графа $T=\{n \in \mathbb{Z}\}-$ множества всех целых чисел с градуировкой $n \rightarrow|n| \in \mathbb{N}$ ) «паскализация» есть граф Паскаля (бесконечный треугольник Паскаля). Как показано в [5], граф ветвления бесконечномерной алгебры Брауэра есть паскализация графа Юнга; другой пример паскализации см. в [15].

2) Заметим, что от выбора начальной вершины в графе Кэли структура динамического графа не зависит, поскольку группа $G$ действует транзитивно на множестве вершин графа $T(G, A)$.

3) В теории динамических систем центральные марковские меры - это меры на марковском компакте с максимальной энтропией. 


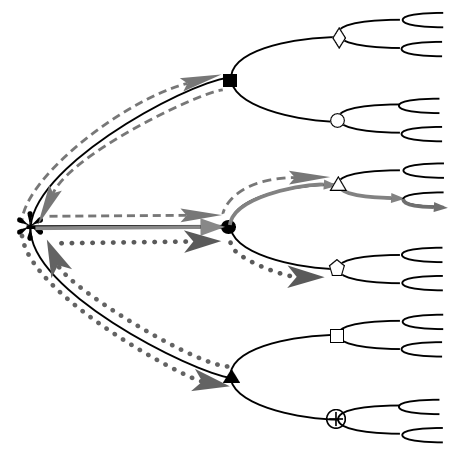

Рис. 1.

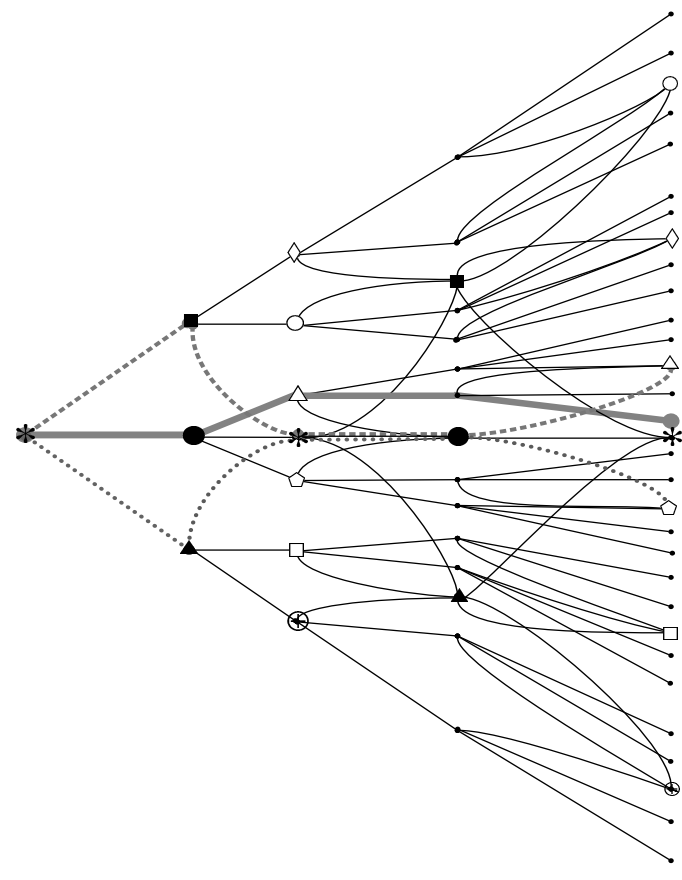

Рис. 2.

Центральные меры образуют выпуклый компакт, являющийся компактным симплексом (см. [3]) в компакте всех мер на пространстве путей. Мера на пространстве путей называется эргодической (или регулярной), если она является экстремальной точкой в этом симплексе. Другое определение эргодичности использует хвостовую фильтрацию. Так называется естественная фильтрация сигма-алгебр $\left\{\mathfrak{A}_{n}\right\}_{n \in \mathbb{N}}$, где $\mathfrak{A}_{n}$ есть сигма-алгебра множеств, описываемых в терминах условий на координаты (вершины) путей с номерами $>n$. Центральная мера $\mu$ эргодична, если пересечение по $n$ последовательности этих сигма-алгебр как сигма-алгебр $\mu$-измеримых множеств есть тривиальная сигмаалгебра, состоящая из множеств нулевой или полной меры.

Теперь мы формулируем нашу основную задачу применительно к задаче о случайных блужданиях на деревьях. Разумеется, постановка задачи имеет смысл для произвольного графа ветвления.

Описать множество $\operatorname{Erg}\left(\mathrm{D}\left(T_{q+1}\right)\right)$ всех эргодических центральных мер на компакте $\mathscr{P}\left(\mathrm{D}\left(T_{q+1}\right)\right)$ бесконечных путей графа $\mathrm{D}\left(T_{q+1}\right)$ или, в других терминах, описать на $\mathscr{P}\left(\mathrm{D}\left(T_{q+1}\right)\right)$ все эргодические марковские меры с максимальной энтропией.

Множество $\operatorname{Erg}\left(\mathrm{D}\left(T_{q+1}\right)\right.$ мы называем в данной ситуации границей-выход. Поскольку рассматриваются марковские меры, то слово «описать» означает в данном случае найти переходные вероятности этих марковских иепей. Именно это и будет сделано.

Отметим отличие этой задачи от задачи нахождения границы ПуассонаФюрстенберга для простых случайных блужданий на группах. В последней фиксируется марковская цепь, отвечающая простому случайному блужданию 
(т. е. мера на путях динамического графа), и требуется найти границу-выход данной марковской цепи (см. [6]). Иначе говоря, требуется представить эту марковскую меру как интеграл по некоторой (гармонической) мере от эргодических центральных мер. Пространство эргодических центральных мер с гармонической мерой и есть граница Пуассона (или граница Пуассона-Фюрстенберга [4]). Она является пространством с мерой. В нашем же случае мы хотим найти все эргодические центральные марковские меры. Ответом служит некоторое топологическое пространство без выделенной меры. Различие видно уже для группы $\mathbb{Z}$ - граница Пуассона здесь тривиальна, а «полная» граница-выход есть интервал.

В этой связи уместно разобрать простейший частный случай нашей задачи, а именно, случай дерева $T_{2}$, т. е. цепи, - он понадобится далее. Как указывалось, граф $\mathrm{D}\left(T_{2}\right)$ есть граф Паскаля, и мы приходим к задаче об описании центральных мер на пространстве путей графа Паскаля. Заметим, что это пространство путей есть пространство всех 0-1-последовательностей, а центральность меры на пространстве путей - это ее инвариантность относительно группы финитных подстановок. Поэтому, применяя теорему де Финетти в нужном варианте, получаем, что эргодическая мера есть мера Бернулли с вероятностью нуля, равной $p$, и с вероятностью единицы, равной $1-p$, а граница-выход для графа Паскаля есть отрезок $[0,1]$; его крайние точки $\{0\}$ и $\{1\}$ отвечают дельта-мерам, сосредоточенным на двух крайних лучах треугольника Паскаля. Правильнее считать, что эта граница есть конус над двумя крайними точками. Заметим, что эргодичность здесь означает не колмогоровский закон 0-1, а так называемый закон Хьюитта-Сэвиджа. Как уже было отмечено, граница Пуассона здесь тривиальна (одноточечна), а граница-выход есть отрезок $[0,1]$. В этом смысле основной результат статьи об описании границы-выход для произвольного однородного дерева можно рассматривать как еще одно («некоммутативное») обобщение теоремы де Финетти. Задачу о границе-выход для динамического графа Кэли произвольной пары $(G, A)$, где $G$ - группа, а $A-$ множество ее образующих и обратных к ним (см. выше), можно считать аналогом задачи де Финетти о симметричных мерах. По-видимому, во всех групповых примерах имеет место стандартность хвостовой фильтрации в смысле [3] и компактность относительно внутренней метрики; в случае рассматриваемого здесь графа $\mathrm{D}(T)$, где $T$ дерево, это следует из основного результата о границе-выход.

Наиболее важное следствие нашего анализа состоит в наличии фазового перехода; его можно описать двумя эквивалентными способами: 1) фазовый переход объясняется потерей эргодичности центральной меры при изменении скорости сноса, 2) фазовый переход объясняется потерей минимальности собственной функции оператора Лапласа при ее деформации и при сохранении собственного числа. Заметим, что теории собственных функций оператора Лапласа на деревьях, на римановых многообразиях постоянной отрицательной кривизны, на гиперболических группах посвящено много работ (наиболее близкие к нашей теме - это [12], [18]). В этих случаях обычно доказывается формула Пуассона для представления произвольных собственных функций в виде интеграла по минимальным собственным функциям. Однако для нас представляет интерес не просто линейная теория собственных функций, но их связь с упорядочением: рассмотрение положительных собственных функций и их интерпретация как марковских (центральных) мер и приводит к обнаружению эффекта фазового 
перехода. Чрезвычайно интересно, для каких графов, групп (в том числе групп Ли) или однородных пространств этот эффект имеет место. Можно предположить, что это так для некоторых симметрических пространств полупростых групп Ли.

\section{§2. Формулировка результата и комментарии}

Определение. Семейство мер $\lambda_{\omega, r}$. Пусть $q \geqslant 2-$ натуральное число, $T_{q+1}$ есть $(q+1)$-однородное дерево и $\mathrm{D}\left(T_{q+1}\right)$ - динамический граф этого дерева (определение дано выше). Естественную проекцию $\mathrm{D}\left(T_{q+1}\right) \rightarrow T_{q+1}$ обозначим через $\pi$. Пространство концов $\omega$ дерева обозначим через $\partial T_{q+1}$; для $q>1$ оно является канторовским множеством. Если вершины $v$ и $w$ смежны в $\mathrm{D}\left(T_{q+1}\right)$, причем $v$ располагается на уровне с бо́льшим номером, чем $w$, будем говорить, что $w$ следует непосредственно за $v$ и писать $v \prec w$. Ребро $(v, w)$ графа $\mathrm{D}\left(T_{q+1}\right)$ (при $\left.v \prec w\right)$ будем называть $\omega$-направленньмм, если проекция $\pi(w)$ лежит между ${ }^{1)} \pi(v)$ и $\omega$.

Для конца $\omega \in \partial T_{q+1}$ и числа $r \in[0,1]$ обозначим через $\lambda_{\omega, r}$ марковскую меру на пространстве $\mathscr{P}\left(\mathrm{D}\left(T_{q+1}\right)\right)$ всех бесконечных путей в графе $\mathrm{D}\left(T_{q+1}\right), \mathrm{y}$ которой вероятности перехода одинаковы и равны $r$ на всех $\omega$-направленных ребрах, а также одинаковы (и равны $(1-r) / q)$ на всех остальных ребрах.

Очевидно, что все меры $\lambda_{\omega, r}$ центральны.

Теорема 2.1. При $q \geqslant 2$ множество $\operatorname{Erg}\left(\mathrm{D}\left(T_{q+1}\right)\right)$ всех эргодических иентральных мер на компакте $\mathscr{P}\left(\mathrm{D}\left(T_{q+1}\right)\right)$ бесконечных путей в динамическом графе $\mathrm{D}\left(T_{q+1}\right) \quad(q+1)$-однородного дерева $T_{q+1}$ (m.е. граница-выход) совпадает со следующим семейством марковских мер:

$$
\Lambda_{q}:=\left\{\lambda_{\omega, r} \mid \omega \in \partial T_{q+1}, r \in[1 / 2,1]\right\} .
$$

Таким образом, граница-выход гомеоморфна (в слабой топологии) произведению

$$
\partial T_{q+1} \times[1 / 2,1]
$$

Замечания. 1. Однородность. Из теоремы следует, что все эргодические центральные марковские меры на $\mathscr{P}\left(\mathrm{D}\left(T_{q+1}\right)\right)$ являются однородными по времени (в том смысле, что отвечающие им переходные вероятности зависят только от вершин дерева и не зависят от момента посещения вершины), поскольку принадлежат описанному семейству, все элементы которого таковы. Однородность не имеет места, вообще говоря, для произвольных центральных мер.

2. Параметр $r$ просто выражается через скорость $\tau$ стремления траекторий к концу дерева $\omega \in \partial T_{q+1}: \tau=2 r-1$. В данном случае $|\tau|$ совпадает с пределом отношения $d\left(\pi(\varnothing), \pi\left(w_{n}\right)\right) / n$ (скоростью сноса, т. е. ухода траектории $\pi\left(w_{n}\right)$ на бесконечность). При $r>1 / 2$ скорость $\tau$ характеризуется также как скорость стабилизации - предел отношения $s(n) / n$, где $s(n)$ есть максимальный номер вершины на луче $[\pi(\varnothing), \omega\rangle$ среди всех вершин этого луча, которые больше не встретятся на данном случайном маршруте (см. лемму 3.2 ). Значение $r=1$ отвечает марковской мере, являющейся дельта-мерой на геодезической - детерминированном пути, ведущем из начальной точки в точку границы $\omega$.

1) Любая вершина дерева соединена единственным геодезическим путем с любым концом $\omega$, поэтому для каждой вершины $x \in T_{q+1}$ и конца $\omega \in \partial T_{q+1}$ определена единственная среди смежных с $x$ вершин, лежащая между $x$ и $\omega$. 
3. Как гласит теорема, марковские меры, отвечающие параметру $r \in[0,1 / 2)$, являются центральными, но уже не являются эргодическими; таким образом, в точке $1 / 2$ имеет место фазовый переход типа потери эргодичности; см. далее $\S 6$. Разложение этих неэргодических центральных мер на эргодические компоненты приведено там же.

4. Граница Пуассона-Фюрстенберга содержится в семействе $\Lambda_{q}$ как подмножество

$$
\left\{\lambda_{\omega, q /(q+1)}: \omega \in \partial T_{q+1}\right\} .
$$

5. Представляет интерес рассмотрение адических преобразований путей (аналогичных автоморфизму Паскаля) и хвостовых фильтраций, соответствующих марковским мерам $\lambda_{\omega, r}$. Из наших результатов следует, что эти фильтрации стандартны в смысле работы [3].

\section{§3. Схема доказательства и первые леммы}

Доказательство основного результата работы - теоремы 2.1 - очевидным образом сводится к доказательству следующих двух утверждений.

I. Всякая эргодическая центральная мера на компакте $\mathscr{P}\left(\mathrm{D}\left(T_{q+1}\right)\right)$ имеет вид $\lambda_{\omega, r}$ (предложение 4.1).

II. Мера вида $\lambda_{\omega, r}$ является эргодической, если и только если $r \in[1 / 2,1]$ (предложение 5.1).

Доказательство утверждения I в существенной степени опирается на теорему о сходимости почти всюду мартингалов (иными словами, индивидуальную эргодическую теорему), применяемую к хвостовой фильтрации на пространстве путей графа.

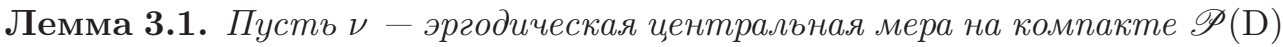
бесконечных путей динамического графа $\mathrm{D}$, a $p_{\nu}$ - соответствующая ей система переходных вероятностей на ребрах графа D. Тогда

(i) для каждого конечного пути $R=\left(\varnothing=v_{0}, \ldots, v_{k}\right)$ в $\mathrm{D}$ и для почти всякого по мере $\nu$ ( $\nu$-n.в.) бесконечного пути $\left(w_{i}\right)_{i \in \mathbb{N}_{0}}$ в $\mathrm{D}$ последовательность ${ }^{1)}$ $\operatorname{dim}\left(v_{k}, w_{i}\right) / \operatorname{dim}\left(w_{i}\right), i \in \mathbb{N}_{0}$, стремится $к$ пределу, причем

$$
\lim _{i \rightarrow \infty} \frac{\operatorname{dim}\left(v_{k}, w_{i}\right)}{\operatorname{dim}\left(w_{i}\right)}=p_{\nu}\left(v_{0}, v_{1}\right) \cdots p_{\nu}\left(v_{k-1}, v_{k}\right) ;
$$

(ii) если $(v, x)$ - ребро в $\mathrm{D}$, то для $\nu$-n.в. пути $\left(w_{i}\right)_{i \in \mathbb{N}_{0}}$ в $\mathrm{D}$ последовательность $\operatorname{dim}\left(x, w_{i}\right) / \operatorname{dim}\left(v, w_{i}\right), i \in \mathbb{N}_{0}$, стремится $к$ пределу, причем

$$
\lim _{i \rightarrow \infty} \frac{\operatorname{dim}\left(x, w_{i}\right)}{\operatorname{dim}\left(v, w_{i}\right)}=p_{\nu}(v, x) .
$$

Определение. Назовем типичным по отношению к заданной эргодической мере $\nu$ путь в графе $\mathrm{D}\left(T_{q+1}\right)$, для которого условия леммы 3.1 выполняются для каждого конечного пути и каждого ребра графа; очевидно, что множество типичных путей имеет полную $\nu$-меру.

Определение. Говорят, что последовательность $\left(x_{i}\right)_{i \in \mathbb{N}}$ в дереве $T_{q+1}$ cходится к точке $\omega \in \partial T_{q+1}$, если для некоторой (и, значит, для любой) вершины

1) Мы пользуемся принятыми в теории графов ветвления обозначениями: число путей, ведущих из вершины $x$ в вершину $y$, обозначается через $\operatorname{dim}(x, y)(\operatorname{a} \operatorname{dim}(\varnothing, y)-$ через $\operatorname{dim}(y))$. Эти обозначения объясняются связью с алгебраической интерпретацией диаграмм Браттели. 
$v \in T_{q+1}$ длина общей части $\left[v, x_{i}\right] \cap[v, \omega\rangle$ геодезического сегмента (пути) $\left[v, x_{i}\right]$ из $v$ в $x_{i}$ и луча $[v, \omega\rangle$ из $v$ в $\omega$ стремится к бесконечности с ростом $i$.

Если проекция в $T_{q+1}$ бесконечного пути в $\mathrm{D}\left(T_{q+1}\right)$ сходится к точке $\omega \in$ $\partial T_{q+1}$, будем говорить, что и сам путь сходится $\kappa \omega$.

Лемма 3.2. Если иентральная мера $\nu$ на компакте путей $\mathscr{P}\left(\mathrm{D}\left(T_{q+1}\right)\right)$ является эргодической, то в дт

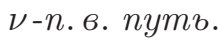

Доказательство. Нетрудно проверить, что отвечающее мере $\nu$ случайное блуждание в $T_{q+1}$ невозвратно: какова бы ни была вершина $v \in T_{q+1}$, почти всякий (по мере $\nu$ ) путь посещает множество $\pi^{-1}(v)$ не более конечного числа раз. Следовательно, поскольку $T_{q+1}$ - дерево, $\nu$-п. в. путь сходится к некоторой точке в $\partial T_{q+1}$. Остается заметить, что в силу эргодичности предельная точка у почти всех путей должна быть одной и той же ${ }^{1)}$.

\section{§4. Однородность}

Настоящий параграф целиком посвящен доказательству следующего предложения 4.1, составляющего первую часть теоремы 2.1.

Предложение 4.1. Всякая эргодическая иентралъная мера на компакте $\mathscr{P}\left(\mathrm{D}\left(T_{q+1}\right)\right)$ бесконечных путей в динамическом графе $\mathrm{D}\left(T_{q+1}\right)$ есть мера вида $\lambda_{\omega, r}$ для некоторых $\omega \in \partial T_{q+1}, r \in[0,1]$, m.е. для кажсдй эргодической иентральной меры $\nu$ найдется такая точка $\omega \in \partial T_{q+1}$, что отвечаяице мере $\nu$ переходные вероятности одинаковы на всех $\omega$-направленных ребрах, а такэсе одинаковы на всех остальных ребрах.

Доказательство. Разберем сначала случай невырожденной ${ }^{2)}$ эргодической меры $\nu$.

В силу леммы 3.2 мера $\nu$ определяет точку $\omega \in \partial T_{q+1}$, к которой сходятся $\nu$-п. в. пути. Покажем, что $\nu=\lambda_{\omega, r}$ для некоторого $r \in[0,1]$.

Сначала заметим, что в каждой вершине $v \in \mathrm{D}\left(T_{q+1}\right)$ переходная вероятность $p_{\nu}$ принимает одинаковые значения на множестве тех исходящих из $v$ ребер, которые не являются $\omega$-направленными. Действительно, если $\left(v, v^{\prime}\right)$ и $\left(v, v^{\prime \prime}\right)-$ два не являющихся $\omega$-направленными ребра, а $\left(w_{i}\right)_{i \in \mathbb{N}_{0}}-$ путь, типичный для $\nu$ и сходящийся к $\omega$, то, как следует из соображений симметрии, для всех достаточно больших $j \in \mathbb{N}$ выполняется равенство $\operatorname{dim}\left(v^{\prime}, w_{j}\right)=\operatorname{dim}\left(v^{\prime \prime}, w_{j}\right)$, откуда в силу типичности пути $\left(w_{i}\right)_{i \in \mathbb{N}_{0}}$ по лемме 3.1 вытекает, что

$$
p_{\nu}\left(v, v^{\prime}\right)=\lim _{j \rightarrow \infty} \frac{\operatorname{dim}\left(v^{\prime}, w_{j}\right)}{\operatorname{dim}\left(v, w_{j}\right)}=\lim _{j \rightarrow \infty} \frac{\operatorname{dim}\left(v^{\prime \prime}, w_{j}\right)}{\operatorname{dim}\left(v, w_{j}\right)}=p_{\nu}\left(v, v^{\prime \prime}\right) \text {. }
$$

Поскольку из каждой вершины динамического графа $\mathrm{D}\left(T_{q+1}\right)$ исходит в точности одно $\omega$-направленное ребро, в силу доказанного факта принадлежность меры $\nu$ к семейству $\left\{\lambda_{\omega, r}: r \in[0,1]\right\}$ вытекает из следующего утверждения.

1) Однородность по времени здесь не предполагалась, ее наличие для эргодических мер будет доказано в следующем параграфе.

2) Мера $\nu$ на компакте $\mathscr{P}\left(\mathrm{D}\left(T_{q+1}\right)\right)$ называется невырожденной, если для любого конечного пути $P$ в $\mathrm{D}\left(T_{q+1}\right)$ мера $\nu(P)$ множества бесконечных путей, начинающихся с $P$, положительна. (Другими словами, мера невырожденна, если отвечающие ей переходные вероятности не обращаются в 0 ни на одном из ребер.) 
Соответствующие мере $\nu$ переходные вероятности $p_{\nu}$ одинаковы на всех $\omega$-направленных ребрах.

В силу связности графа $\mathrm{D}\left(T_{q+1}\right)$ достаточно проверить лишь, что переходные вероятности $p_{\nu}$ совпадают на $\omega$-направленных ребрах, исходящих из смежных вериин.

Пусть $a \prec b$ - смежные вершины в $\mathrm{D}\left(T_{q+1}\right)$. Для вершины $v$ будем обозначать через $v_{\omega}$ концевую вершину $\omega$-направленного ребра, исходящего из $v$. Нам нужно показать, что $p_{\nu}\left(a, a_{\omega}\right)=p_{\nu}\left(b, b_{\omega}\right)$.

Пусть, как и выше, $\left(w_{i}\right)_{i \in \mathbb{N}_{0}}$ - типичный путь, сходящийся к $\omega$. Тогда в силу леммы 3.1 получаем, что

$$
\lim _{i \rightarrow \infty} \frac{\operatorname{dim}\left(a_{\omega}, w_{i}\right)}{\operatorname{dim}\left(a, w_{i}\right)}=p_{\nu}\left(a, a_{\omega}\right), \quad \lim _{i \rightarrow \infty} \frac{\operatorname{dim}\left(b_{\omega}, w_{i}\right)}{\operatorname{dim}\left(b, w_{i}\right)}=p_{\nu}\left(b, b_{\omega}\right) .
$$

Покажем, что найдется бесконечно много чисел $j \in \mathbb{N}$, таких, что

$$
\operatorname{dim}\left(a, w_{j}\right)=\operatorname{dim}\left(b, w_{j+1}\right), \quad \operatorname{dim}\left(a_{\omega}, w_{j}\right)=\operatorname{dim}\left(b_{\omega}, w_{j+1}\right),
$$

откуда в силу (2) сразу последует искомое равенство $p_{\nu}\left(a, a_{\omega}\right)=p_{\nu}\left(b, b_{\omega}\right)$.

Заметим, что $\operatorname{dim}(x, y)$ - число путей из вершины $x$ в вершину $y$ в графе $\mathrm{D}\left(T_{q+1}\right)$ - совпадает с числом маршрутов длины $|L(x)-L(y)|$ между вершинами $\pi(x)$ и $\pi(y)$ в дереве $T_{q+1}$ (здесь и далее $L(z)$ - номер уровня, на котором находится вершина $z)$. Заметим, кроме того, что в однородном дереве количество маршрутов заданной длины между двумя вершинами определяется лишь расстоянием между ними. Наконец, заметим, что поскольку по построению $L(b)=L(a)+1, L\left(v_{\omega}\right)=L(v)+1$ и $L\left(w_{i+1}\right)=L\left(w_{i}\right)+1$, для любого $i \in \mathbb{N}$ мы имеем (ср. с (3))

$$
L(a)-L\left(w_{i}\right)=L(b)-L\left(w_{i+1}\right), \quad L\left(a_{\omega}\right)-L\left(w_{i}\right)=L\left(b_{\omega}\right)-L\left(w_{i+1}\right) .
$$

Отсюда следует, что соотношения (3) будут выполняться для всякого $j \in \mathbb{N}$, при котором выполняются соотношения

$$
d\left(\pi(a), \pi\left(w_{j}\right)\right)=d\left(\pi(b), \pi\left(w_{j+1}\right)\right), \quad d\left(\pi\left(a_{\omega}\right), \pi\left(w_{j}\right)\right)=d\left(\pi\left(b_{\omega}\right), \pi\left(w_{j+1}\right)\right) .
$$

Итак, нам остается доказать, что найдется бесконечно много чисел $j \in \mathbb{N}$, для которых выполняются соотношения (5).

Ситуация разбивается на два случая:

(i) $b=a_{\omega}$, т. е. ребро $(a, b)$ является $\omega$-ориентированным,

(ii) $b \neq a_{\omega}$, т. е. ребро $(a, b)$ не является $\omega$-ориентированным.

Поскольку маршрут $\left(\pi\left(w_{i}\right)\right)_{i \in \mathbb{N}_{0}}$ следует по смежным вершинам дерева $T_{q+1}$, для любых $u \in T_{q+1}$ и $i \in \mathbb{N}$

$$
d\left(u, \pi\left(w_{i+1}\right)\right)=d\left(u, \pi\left(w_{i}\right)\right) \pm 1
$$

Поскольку $\left(\pi\left(w_{i}\right)\right)_{i \in \mathbb{N}_{0}}$ к тому же сходится к $\omega$, в случае $(\mathrm{i})$ соотношения $(5)$ выполняются всякий раз, когда $j$ достаточно велико и

$$
d\left(\pi(a), \pi\left(w_{j+1}\right)\right)=d\left(\pi(a), \pi\left(w_{j}\right)\right)+1,
$$

а в случае (ii) они выполняются всякий раз, когда $j$ достаточно велико и

$$
d\left(\pi(a), \pi\left(w_{j+1}\right)\right)=d\left(\pi(a), \pi\left(w_{j}\right)\right)-1 .
$$

Индексов $j$, при которых выполняется (6), найдется бесконечно много уже потому, что $\left(\pi\left(w_{i}\right)\right)_{i \in \mathbb{N}_{0}}$ следует по смежным вершинам и сходится к $\omega$. Индексов, при которых выполняется (7), также найдется бесконечно много, потому 
что в противном случае, начиная с некоторого $i$, все ребра $\left(w_{i}, w_{i+1}\right)$ были бы $\omega$-направленными, а это противоречит (как следует из леммы 3.1) предположению о невырожденности меры $\nu$.

Перейдем к случаю вырожденной эргодической меры $\nu$ - когда в $\mathrm{D}\left(T_{q+1}\right)$ имеются $\nu$-недостижимые, т. е. которых нельзя достичь с положительной $\nu$-вероятностью, вершины и переходная вероятность $p_{\nu}$ на некоторых ребрах обращается в 0. Мы докажем, что $\nu$ имеет вид $\lambda_{\omega, 1}$.

Действительно, если некоторая вершина $v$ является $\nu$-недостижимой, то в силу центральности меры $\nu$ недостижимыми являются и все вершины, следующие за $v$ (т. е. вершины, лежащие на уровнях с бо́льшими номерами, чем $v$, и соединенные с $v$ путями). Вспомним, кроме того, что в силу леммы 3.2 эргодическая мера $\nu$ определяет некоторую точку $\omega \in \partial T_{q+1}, \mathrm{k}$ которой сходятся $\nu$-п. в. пути. Однако множество сходящихся к $\omega$ путей, не проходящих ни через вершину $v$, ни через вершины, следующие за $v$, не более чем счетно. Таким образом, $\nu$ сосредоточена на не более чем счетном множестве путей. Отсюда в силу эргодичности меры $\nu$ следует, что она сосредоточена на одном пути, а в силу центральности, - что этот путь образует одноточечный класс хвостового разбиения. Такой путь для $\omega$ единствен, а мера, сосредоточенная на таком пути - это мера $\lambda_{\omega, 1}$.

\section{§5. Список эргодических центральных мер и разложение по ним неэргодических мер}

Для завершения доказательства теоремы 2.1 остается доказать следующее предложение.

Предложение 5.1. Мера вида $\lambda_{\omega, r}$ является эргодической, если и толъко если $r \in[1 / 2,1]$.

Доказательство. Доказательство неэргодичности при $r<1 / 2$. Напомним, что меру на путях графа $\mathrm{D}(T)$ можно рассматривать как меру, отвечающую марковскому процессу. Пусть $\omega \in \partial T_{q+1}$ и $r \in[0,1 / 2)$. Рассмотрим меру $\lambda_{\omega, r}$ и соответствующий марковский процесс на $T_{q+1}$. Этот процесс однороден по времени по определению меры $\lambda_{\omega, r}$; его переходные вероятности принимают значение $r$ на ориентированном ребре $(x, y)$, если $y$ лежит между $x$ и $\omega$, и значение $(1-r) / q$ в противном случае.

Мы видим, что п.в. траектория процесса сходится к некоторой точке из $\partial T_{q+1} \backslash\{\omega\}$, поскольку удаляется от $\omega$ по $\omega$-орисферам со сносом $1-2 r$ и движется по смежным вершинам. Отсюда следует, что распределение на $\partial T_{q+1} \backslash\{\omega\}$ пределов траекторий этого марковского процесса задается непрерывной мерой, поскольку и сам марковский процесс, и, значит, эта предельная мера на $\partial T_{q+1} \backslash\{\omega\}$ инвариантны по отношению к автоморфизмам дерева $T_{q+1}$, неподвижным на луче $\left[\pi\left(v_{0}\right), \omega\right\rangle$, а группа таких автоморфизмов не имеет конечных орбит в $\partial T_{q+1} \backslash\{\omega\}$.

Полученная непрерывность распределения предельных точек в $\partial T_{q+1}$ и показывает, что $\lambda_{\omega, r}$ не является эргодической, поскольку из леммы 3.2 следует, что почти все траектории марковского процесса в $T_{q+1}$, соответствующего эргодической центральной мере, сходятся к одной и той же точке в $\partial T_{q+1}$. 
Доказательство эргодичности при $r \geqslant 1 / 2^{1)}$. Для доказательства достаточно заметить следующее. Во-первых, доказывается, что почти всякий путь по мере $\lambda_{\omega, r}$ при $r \geqslant 1 / 2$ стремится к $\omega$ со скоростью $2 r-1$ (см. лемму 5.2 ниже). С другой стороны, всякая неэргодическая мера может быть единственным образом разложена в интеграл по эргодическим мерам, которые по доказанному содержатся среди мер семейства $\Lambda_{q}=\left\{\lambda_{\omega, r} \mid \omega \in \partial T_{q+1}, r \in[1 / 2,1]\right\}$. Но мера $\lambda_{\omega_{0}, r_{0}} \in \Lambda_{q}$ не может быть представлена как интеграл по некоторой мере на $\Lambda_{q}$, не являющейся дельта-мерой в точке $\left(\omega_{0}, r_{0}\right)$, а сосредоточенной на некотором множестве параметров $\omega, r$, потому что в этом случае пределы почти всех по мере $\lambda_{\omega_{0}, r_{0}}$ путей и их скорости сходимости к пределу были бы отличны соответственно от $\omega_{0}$ и/или $2 r_{0}-1$, с которыми они должны в действительности совпадать для почти всех путей.

Лемма 5.2. Предположим, что $\omega \in \partial T_{q+1} u r \in[1 / 2,1] . B$ дереве $T_{q+1}$ вершину луча $[\pi(\varnothing), \omega\rangle$, находяшуюся на расстоянии $k \in \mathbb{N}_{0}$ от начальной вершины $\pi(\varnothing)$, обозначим через $v_{k}$. Тогда почти всякий по мере $\lambda_{\omega, r}$ путь $\left(w_{i}\right)_{i \in \mathbb{N}_{0}}$ сxодится $\kappa \omega u$

$$
\frac{d\left(\pi\left(w_{n}\right), v_{\lfloor(2 r-1) n\rfloor}\right)}{n} \stackrel{n \rightarrow \infty}{\longrightarrow} 0 .
$$

Доказательство. Доказательство удобно построить на базе проекции

$$
\mathrm{D}\left(T_{q+1}\right) \stackrel{\pi}{\rightarrow} T_{q+1} \stackrel{-h_{\omega, v_{0}}}{\longrightarrow} \mathbb{Z},
$$

где $h_{\omega, v_{0}}$ есть орифункция ${ }^{2}$ на $T_{q+1}$, стремящаяся к $-\infty$ на лучах, представляющих $\omega$, и принимающая нулевое значение в точке $v_{0}=\pi(\varnothing)$. Эта проекция переводит меру $\lambda_{\omega, r}$ в случайное блуждание на $\mathbb{Z}$ с переходными вероятностями

$$
p(z, z+1)=r, \quad p(z, z-1)=1-r .
$$

Ключевым здесь является следующее наблюдение. Всякий раз, когда маршрутпроекция $\left(-h_{\omega, v_{0}}\left(\pi\left(w_{n}\right)\right)\right)_{n \in \mathbb{N}_{0}}$ обновляет свой максимум в $\mathbb{Z}$, точка $\pi\left(w_{n}\right)$ оказывается на луче $[\pi(\varnothing), \omega\rangle$. Детали мы здесь не приводим.

Приведем явную формулу разложения на эргодические компоненты.

Формула разложения неэргодической меры $\boldsymbol{\lambda}_{\boldsymbol{\omega}, \boldsymbol{r}}$. При $r<1 / 2$ центральная мера $\lambda_{\omega_{0}, r}$ разлагается на эргодические компоненты $\lambda_{\omega, 1-r}$ :

$$
\lambda_{\omega_{0}, r}=\int_{\partial T_{q+1}} \lambda_{\omega, 1-r} \cdot \rho_{\omega_{0}, r}(d \omega) .
$$

Распределение $\rho_{\omega_{0}, r}$ абсолютно непрерывно относительно гармонической меры $\theta=\theta_{v_{0}}$ на $\partial T_{q+1}$, симметричной по отношению к начальной вершине $v_{0}=\pi(\varnothing)$.

1) Можно было бы для доказательства эргодичности мер $\lambda_{\omega, r}, r \geqslant 1 / 2$, использовать энтропийный критерий из [6], который применим и в этой ситуации, но мы используем то, что запас мер, содержащий все эргодические меры, уже найден и остается лишь отобрать те из них, которые не являются усреднениями других мер. Заметим, что стандартность фильтрации, когда она есть, дает мощный критерий эргодичности.

2) Орифункцией на счетном метрическом пространстве $(X, d)$ называется всякая неограниченная снизу функция, являющаяся поточечным пределом функций вида $d\left(x, x_{0}\right)+C$, где $x_{0} \in X$ и $C \in \mathbb{R}$. 
Производная Радона-Никодима $d \rho_{\omega_{0}, r} / d \theta$ задается формулой

$$
\frac{d \rho_{\omega_{0}, r}}{d \theta}(\omega)=\frac{(1-2 r)(q+1)}{q-q r-r}\left(\frac{q r}{1-r}\right)^{\ell\left(\omega_{0}, \omega\right)},
$$

где $\ell\left(\omega, \omega_{0}\right)$ - длина общей части лучей $\left[v_{0}, \omega\right\rangle$ и $\left[v_{0}, \omega_{0}\right\rangle$, а выражение $0^{0}$, возникающее при $r=\ell\left(\omega_{0}, \omega\right)=0$, следует интерпретировать как 1 .

Для вывода формулы (10) мы пользуемся тем, что упомянутая выше проекция (8) переводит меру $\lambda_{\omega, r}$ в случайное блуждание на $\mathbb{Z}$ с переходными вероятностями (9), а при $r<1 / 2$ доля траекторий такого блуждания, достигающих точки $k \in \mathbb{N}_{0} \subset \mathbb{Z}$, но не достигающих $k+1$, составляет

$$
\left(\frac{r}{1-r}\right)^{k}-\left(\frac{r}{1-r}\right)^{k+1}
$$

\section{§6. Фазовый переход, граница Мартина}

В этом параграфе мы обсудим интерпретацию упомянутого выше фазового перехода, а также свойства границы Мартина графа $\mathrm{D}\left(T_{q+1}\right)$.

Как мы видели, при прохождении параметра $r$ через значение $1 / 2$ меры $\lambda_{\omega, r}$ теряют эргодичность. При критическом значении эргодичность сохраняется, но поведение траекторий меняется. При $r>1 / 2$ почти все по мере $\lambda_{\omega, r}$ пути стремятся к пределу $\omega$ с линейной (по $n$ ) скоростью $2 r-1$. При $r=1 / 2$ почти все пути также стремятся к $\omega$, но уже с сублинейной скоростью; в этом случае поведение путей заслуживает более подробного рассмотрения, которое здесь не проводится.

Для того чтобы дать основную интерпретацию фазового перехода, установим соответствие между семейством мер $\lambda_{\omega, r}$ и специальным семейством собственных функций оператора Лапласа на однородном дереве $T_{q+1}$.

Вспомним сначала, что на однородном дереве $T_{q+1}$ множество $\mathscr{H}_{\text {min }}$ минимальных положительных гармонических (т. е. инвариантных относительно оператора Лапласа) функций совпадает с семейством функций вида $q^{-h(v)}$, где $h(v)$ - любая орифункция на $T_{q+1}$. Как нетрудно проверить, при любом (вещественном или комплексном) $\alpha$ степень $\left(q^{-h(v)}\right)^{\alpha}=q^{-\alpha h(v)}$ минимальной гармонической функции $q^{-h(v)}$ является собственной функцией оператора Лапласа с собственным числом

$$
s_{\alpha}=\frac{q^{\alpha}+q^{1-\alpha}}{q+1} .
$$

С другой стороны, каждая положительная собственная функция оператора Лапласа на базовом графе определяет центральную марковскую меру на пространстве путей соответствующего динамического графа: переходная вероятность $p_{\mu}$ центральной меры $\mu=\mu_{f}$, отвечающей собственной функции $f$ с собственным значением $s$, на ребре $(v, w)$ с $v \prec w$ задается формулой

$$
p_{\mu}(v, w)=\frac{f(\pi(w))}{f(\pi(v))(q+1) s} .
$$

Формула (11) дает биекцию между нормированными положительными собственными функциями оператора Лапласа на $T_{q+1}$ и теми невырожденными центральными мерами, которые, будучи марковскими на компакте путей в 
$\mathrm{D}\left(T_{q+1}\right)$, однородны по времени. При этой биекции минимальные собственные функции соответствуют эргодическим центральным мерам.

Если $\omega$ - конец дерева $T_{q+1}$ и $h_{\omega}$ есть орифункция на $T_{q+1}$, стремящаяся к $-\infty$ на лучах, представляющих $\omega$, а $\alpha \in \mathbb{R}$, то отображение (11) переводит положительную собственную функцию $q^{-\alpha h_{\omega}(v)}$ в центральную меру $\lambda_{\omega, r_{\alpha}}$, где $\mathrm{e}^{1)}$

$$
r_{\alpha}=\frac{1}{1+q^{1-2 \alpha}} .
$$

Тем самым для любого фиксированного $\omega_{0}$ из $\partial T_{q+1}$ установлено соответствие $r \leftrightarrow \alpha$ между мерами $\lambda_{\omega_{0}, r}$, точнее параметрами $r \in(0,1)$, и собственными функциями $q^{-\alpha h_{\omega_{0}}(v)}$, т. е. параметрами $\alpha \in \mathbb{R}$. Поскольку формула $(11)$ дает биекцию между минимальными (нормированными положительными собственными) функциями и эргодическими (центральными марковскими) мерами, в силу предложения 5.1 собственная функция $q^{-\alpha h_{\omega}(v)}$ минимальна, если и только если $\alpha \in[1 / 2,+\infty)$.

Теперь мы можем описать наш фазовый переход не только в терминах эргодичности мер, но и в терминах собственных функций. При переходе показателя $\alpha$ через $1 / 2$ степень $q^{-\alpha h(v)}=\left(q^{-h(v)}\right)^{\alpha}$ гармонической функции $q^{-h(v)}$ по-прежнему остается собственной функцией оператора Лапласа, но перестает быть минимальной, поскольку только минимальным отвечают эргодические меры. Эта интерпретация ближе к обычным моделям фазовых переходов, поскольку параметр $\alpha$ стал здесь просто показателем степени. Авторам неизвестно, встречались ли ранее естественные алгебраические модели фазовых переходов такого рода.

В заключение отметим одно следствие о границе Мартина, вытекающее из наших рассмотрений. Мы упоминали об интерпретации классической конструкции границы Мартина в терминах графа ветвления (диаграммы Браттели) см. [13], [14], [3]. В работе [3] объяснено, как определить границу Мартина геометрическим способом в рамках теории проективных пределов конечномерных симплексов. Граница Мартина вкладывается в симплекс центральных марковских мер, а ее минимальная часть отождествляется при этом с множеством экстремальных точек симплекса (границей-выход).

В случае графа $\mathrm{D}\left(T_{q+1}\right)$ его граница Мартина $\mathscr{M}=\mathscr{M}\left(\mathrm{D}\left(T_{q+1}\right)\right)$ включает границу-выход $\mathscr{M}_{0}=\operatorname{Erg}\left(\mathrm{D}\left(T_{q+1}\right)\right)$ собственным образом: возникающее дополнительное множество $\mathscr{M}^{\prime}=\mathscr{M} \backslash \mathscr{M}_{0}$ образовано счетным множеством изолированных точек, элементы которого находятся во взаимно однозначном соответствии с вершинами дерева $T_{q+1}$. Указанное соответствие $\sigma: T_{q+1} \rightarrow \mathscr{M}^{\prime}$ продолжается до непрерывного вложения $T_{q+1} \cup \partial T_{q+1} \rightarrow \mathscr{M}$, переводящего границу $\partial T_{q+1}$ в край $\partial T_{q+1} \times\{1 / 2\}$. Соответствующая вершине $v \in T_{q+1}$ точка $\sigma(v) \in \mathscr{M}^{\prime}$ является предельной для множества $\pi^{-1}(v)$ в $\mathrm{D}\left(T_{q+1}\right)$.

Отметим, что для нахождения границы Мартина необходимы прямые вычисления, касающиеся степеней сверток или подсчета маршрутов заданной длины между вершинами. Число маршрутов длины $n$, соединяющих две находящиеся на расстоянии $k \in \mathbb{N}_{0}$ вершины в $(q+1)$-однородном дереве $T_{q+1}$ при $n \geqslant k$ и

1) При $\alpha=1 / 2$ получаем $r_{1 / 2}=1 / 2$. 
четном $n-k$ задается формулой (см. также [16], [7], [17])

$$
S_{q}(n, k)=S_{q}(n, k+2)+q^{(n-k) / 2} L(n, k)=\sum_{t \in\{k, k+2, \ldots, n\}} q^{(n-t) / 2} L(n, t),
$$

где

$$
L(n, t)=C_{n}^{(n-t) / 2}-C_{n}^{(n-t) / 2-1}=\frac{t+1}{n+1} C_{n+1}^{(n-t) / 2} .
$$

Этот пример с границей Мартина графа $\mathrm{D}\left(T_{q+1}\right)$ дает ответ (как и ожидалось, отрицательный) на вопрос, поставленный в [3]: можно ли описать границу Мартина в терминах самого предельного симплекса? Действительно, если

$$
\Sigma_{0} \stackrel{\pi_{1,0}}{\longleftarrow} \Sigma_{1} \stackrel{\pi_{2,1}}{\longleftarrow} \Sigma_{2} \leftarrow \cdots \leftarrow \Sigma_{n} \stackrel{\pi_{n+1, n}}{\longleftarrow} \Sigma_{n+1} \leftarrow \cdots \Sigma_{\infty}=\operatorname{Inv}\left(\mathrm{D}\left(T_{q+1}\right)\right)
$$

есть отвечающий графу $\mathrm{D}\left(T_{q+1}\right)$ проективный предел, то у прореженной аппроксимации

$$
\Sigma_{0} \stackrel{\pi_{1,0} \circ \pi_{2,1}}{\longleftarrow} \Sigma_{2} \stackrel{\pi_{3,2} \circ \pi_{4,3}}{\longleftarrow} \Sigma_{4} \leftarrow \cdots \Sigma_{\infty}=\operatorname{Inv}\left(\mathrm{D}\left(T_{q+1}\right)\right)
$$

при том же предельном симплексе граница Мартина уже не содержит тех точек множества $\mathscr{M}^{\prime}$, которые соответствуют находящимся на нечетном расстоянии от $v_{0}$ точкам из $T_{q+1}$ - ведь все симплексы, содержащие подходящие вершины, в прореженной последовательности отсутствуют.

Таким образом, понятие границы Мартина в задаче об инвариантных мерах на данном отношении эквивалентности зависит от аппроксимации этого отношения и в этом смысле не является внутренним для этой задачи.

\section{ЛитеРАТУРА}

[1] A. M. Vershik, Intrinsic metric on graded graphs, standardness, and invariant measures, Теория представлений, динамические системы, комбинаторные методы. XXIII, Зап. научн. сем. ПОМИ, 421 (2014), 58-67.

[2] А. М. Вершик, Задача о централъных мерах на пространствах путей градуированных грабов, Функц. анализ и его прил., 48:4 (2014), 26-46.

[3] А. М. Вершик, Оснащенные градуированные графы, проективные предель симплексов и их гранищы, Теория представлений, динамические системы, комбинаторные методы. XXIV, Зап. научн. сем. ПОМИ, 432 (2015), 83-104.

[4] А. М. Вершик, Динамическая теория роста в группах: энтропия, границь, примерьь, УМН, 55:4(334) (2000), 59-128.

[5] А. М. Вершик, П. П. Никитин, Следъ на бесконечнъх алгебрах Брауэра, Функц. анализ и его прил., 40:3 (2006), 3-11.

[6] V. A. Kaimanovich, A. M. Vershik, Random walks on discrete groups, boundary and entropy, Ann. Probab., 11:3 (1983), 457-490.

[7] Р. И. Григорчук, Симметрические случайные блуждания на дискретных групnax, УМН, 32:6(198) (1977), 217-218.

[8] Е. Б. Дынкин, А. А. Юшкевич, Теоремъ и задачи о процессах Маркова, Наука, M., 1967.

[9] Е. Б. Дынкин, Пространство выходов марковского процесса, УМН, 24:4(148) (1969), 89-152.

[10] E. B. Dynkin, Entrance and exit spaces for a Markov process, in: Actes du Congrès International des Mathématiciens (Nice, 1970), Tome 2, Gauthier-Villars, Paris, 1971, 507-512. 
[11] Е. Б. Дынкин, Началъное и финальное поведение траекторий марковских процессов, УМН, 26:4(160) (1971), 153-172.

[12] A. Figà-Talamanca, C. Nebbia, Harmonic Analysis and Representation Theory for Groups Acting on Homogeneous Trees, London Mathematical Society Lecture Note Series, vol. 162, Cambridge University Press, Cambridge, 1991.

[13] S. V. Kerov, The boundary of Young lattice and random Young tableaux, in: DIMACS Ser. Discrete Math. Theoret. Comput. Sci., vol. 24, Amer. Math. Soc., Providence, RI, 1996, 133-158.

[14] S. Kerov, A. Okounkov, G. Olshansky, The boundary of the Young graph with Jack edge multiplicities, Internat. Math. Res. Notices, No. 4, 1998, 173-199.

[15] F. M. Goodman, S. V. Kerov, The Martin boundary of the Young-Fibonacci lattice, J. Algebraic Combin., 11:1 (2000), 17-48.

[16] Б. Левит, С. А. Молчанов, Инвариантные цепи на свободных группах с конечным числом образующих, Вестник Московск. унив., о. 6, 1971, 80-88.

[17] M. Pagliacci, Heat and wave equation on homogeneous trees, Boll. Un. Mat. Ital. Ser. VII, A7:1 (1993), 37-45.

[18] S. Helgason, Eigenspaces of the Laplacian, integral representations and irreducibility, J. Funct. Anal., 17 (1974), 328-353.

[19] Y. Guivar'h, Ji Lizhen, J. C. Taylor, Compactifications of Symmetric Spaces, Progress in Math., vol. 156, Birkhauser, Boston, 1998.

Санкт-Петербургское отделение Математического института им. В. А. Стеклова РАН

Поступила в редакцию Санкт-Петербургский государственный университет

31 марта 2015 г.

Институт проблем передачи информации

им. А. А. Харкевича РАН

e-mail: avershik@gmail.com

Санкт-Петербургское отделение Математического

института им. В. А. Стеклова РАН

e-mail: malyutin@pdmi.ras.ru 